\title{
Complete mucosal repair after endoscopic submucosal enucleation of a gastric submucosal tumor assisted by the clip-with-line method
}

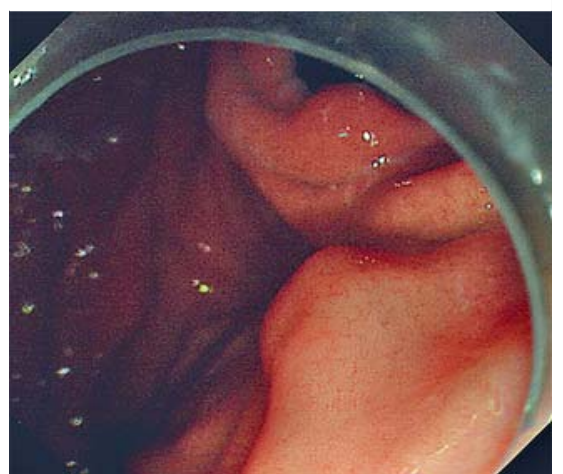

> Fig. 1 Esophagogastroduodenoscopy showing a protrusion in the gastric body of a 30-year-old man admitted to hospital because of dyspepsia.

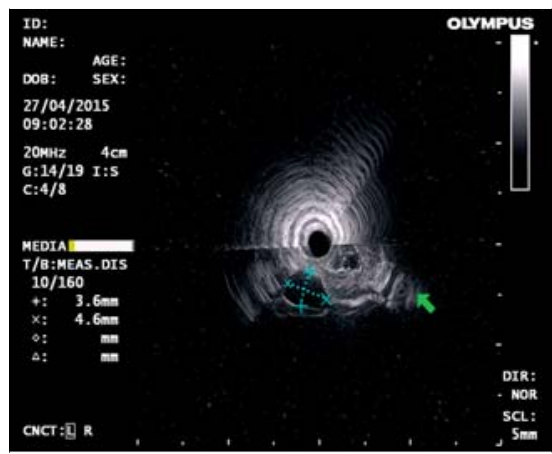

- Fig. 2 Endoscopic ultrasound shows a hypoechoic mass originating from the muscularis propria. Blue crosses show the diameter of the tumor and the green arrow indicates the muscularis propria layer.

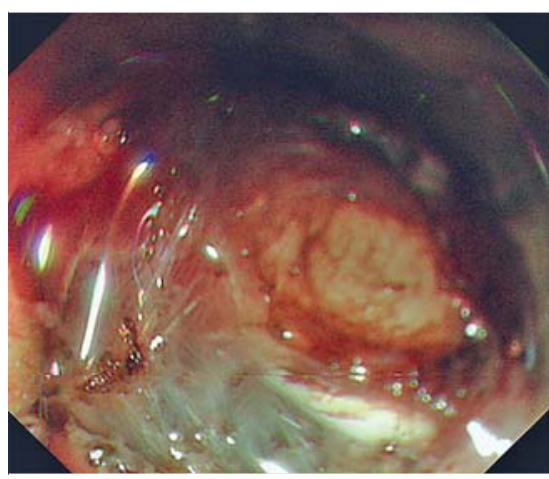

- Fig. 3 Deep semicircumferential mucosal incision exposing the gastric submucosal tumor.
Deep ulcers and even perforations can occur after endoscopic submucosal enucleation (ESE) of gastrointestinal submucosal tumors (SMTs), as most SMTs arise from the muscularis propria layer. If SMTs can be removed by ESE without damaging the overlying normal mucosa, the defects can be completely closed with the remaining normal mucosa. Recently, countertraction has been reported to facilitate resection of gastrointestinal SMTs [1]. In this report, we describe how to use a clip-with-line (CWL) method to facilitate enucleation of an SMT, with the entire overlying normal mucosa preserved to close the defect completely after ESE.

A 30-year-old man was admitted to our hospital because of dyspepsia. A gastric SMT was detected endoscopically (> Fig.1). Subsequently, endoscopic ultrasound was used to show that the SMT originated in the muscularis propria layer ( $\mathbf{F i g . 2}$ ). At the patient's express request, ESE was performed; written informed consent was obtained before treatment. First, after marking and submucosal injection, a semicircumferential mucosal incision was created using a DualKnife from Olympus (Tokyo, Japan). Submucosal dissection exposed the SMT

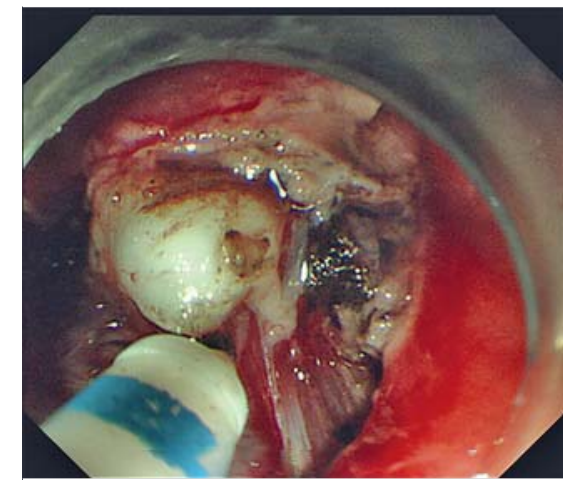

- Fig. 4 The tumor could be clearly visualized when traction was applied.

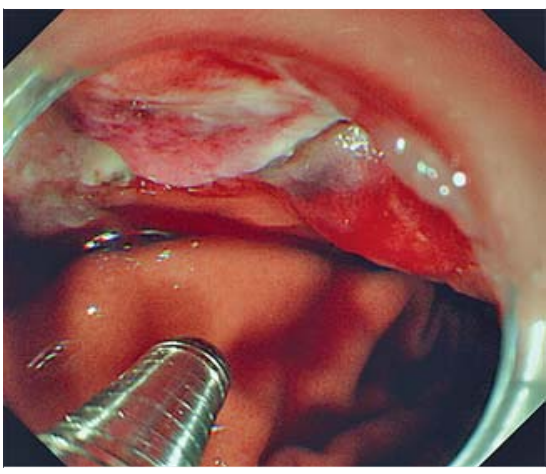

- Fig. 5 After the traction had been released, the conserved mucosa was placed over the wound.

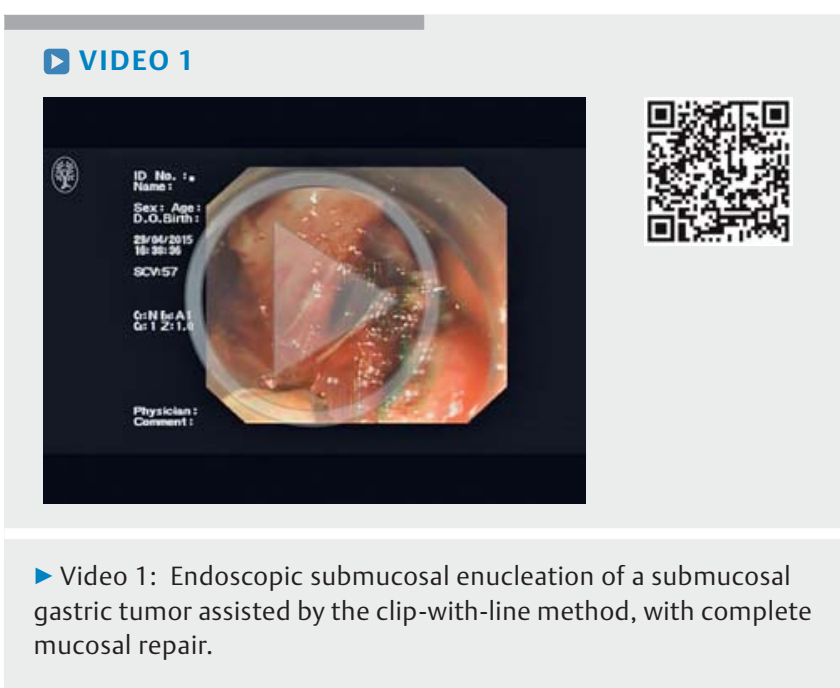


(• Fig.3). The CWL was subsequently attached to the flap of loosened mucosa, to facilitate adequate countertraction and clear visualization when the line was gently pulled out (> Fig.4) [2] The tumor was enucleated from the muscularis propria layer and the overlying normal mucosa remained undamaged. Although a small perforation was seen, the wound was completely closed using the conserved mucosa as follows. The traction was released and the conserved mucosa was applied to the wound by means of the transparent hood attached to the scope ( $\mathbf{F i g} \mathbf{5}$ ). An additional four clips were used to attach the conserved mucosa to the defect $(\triangleright$ Video 1$)$.

This method facilitates ESE and complete mucosal repair, enabling faster mucosal healing with less deformity, and perhaps even perforation repair.

Endoscopy_UCTN_Code_TTT_1AO_2AG
Competing interests

None

The Authors

Yu-qi He, Ai-qin Li, Jian-qiu Sheng, Dong-liang Yu, Kuang-I Fu

Department of Gastroenterology, PLA Army

General Hospital, Beijing, China

Corresponding author

\section{Kuang-I Fu, MD}

Department of Gastroenterology, PLA Army General Hospital, Nanmenchang 5\#, Dongcheng District, Beijing 100700, China, Fax: +86-10-66721168,

fukuangi@hotmail.com

\section{References}

[1] Lu J, Jiao T, Li Y et al. Facilitating retroflexed endoscopic full-thickness resection through loop-mediated or rope-mediated countertraction (with videos). Gastrointest Endosc 2016; 83: $223-228$

[2] Oyama T. Counter traction makes endoscopic submucosal dissection easier. Clin Endosc 2012; 45: 375-378

\section{Bibliography}

DOI http://dx.doi.org/10.1055/s-0042-119037

Endoscopy 2017; 49: E3-E4

(c) Georg Thieme Verlag KG

Stuttgart · New York

ISSN 0013-726X 